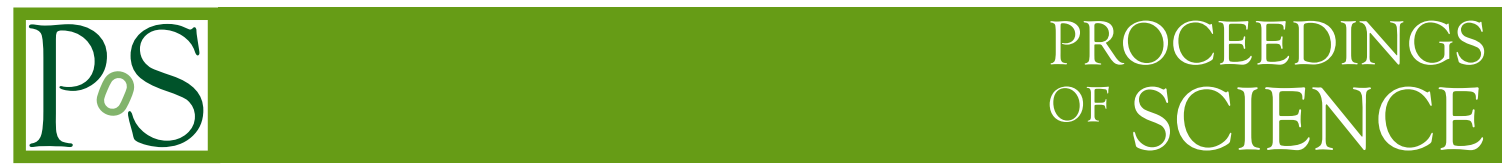

\title{
Particle identification for Belle II
}

\section{Tomokatsu Hayakawa ${ }^{* \dagger}$}

Kobayashi-Maskawa Institute for the Origin of Particles and the Universe, Nagoya University, Japan

E-mail: hayakawa@hepl.phys.nagoya-u.ac.jp

The Belle / KEKB is being upgraded to Belle II / SuperKEKB with the goal of probing new physics beyond the Standard Model (SM). In order to improve particle identification (PID), we will replace Belle PID systems with RICH type sub-detectors, called TOP counter and ARICH. In addition, we are upgrading Belle electromagnetic calorimeter (ECL) and $K_{L}^{0}-\mu$ detector (KLM) to handle the larger beam related backgrounds. In this paper, the construction status of Belle II PID systems is briefly described.

The 15th International Conference on B-Physics at Frontier Machines at the University of Edinburgh, 14 -18 July, 2014

University of Edinburgh, $U K$

\footnotetext{
* Speaker.

${ }^{\dagger}$ for the Belle II Collaboration
} 


\section{Introduction}

The KEK B-factory is being upgraded to SuperKEKB using the same tunnel as KEKB[1]. The SuperKEKB is an asymmetric electron-positron collider and aims to increase the peak luminosity by about a factor 40 by the upgrade based on the Nano-Beam scheme and high beam current. The target luminosity is $8 \times 10^{35} \mathrm{~cm}^{-2} \mathrm{~s}^{-1}$ and this improvement allows us to discover new physics beyond the Standard Model.

\section{Belle II Detector}

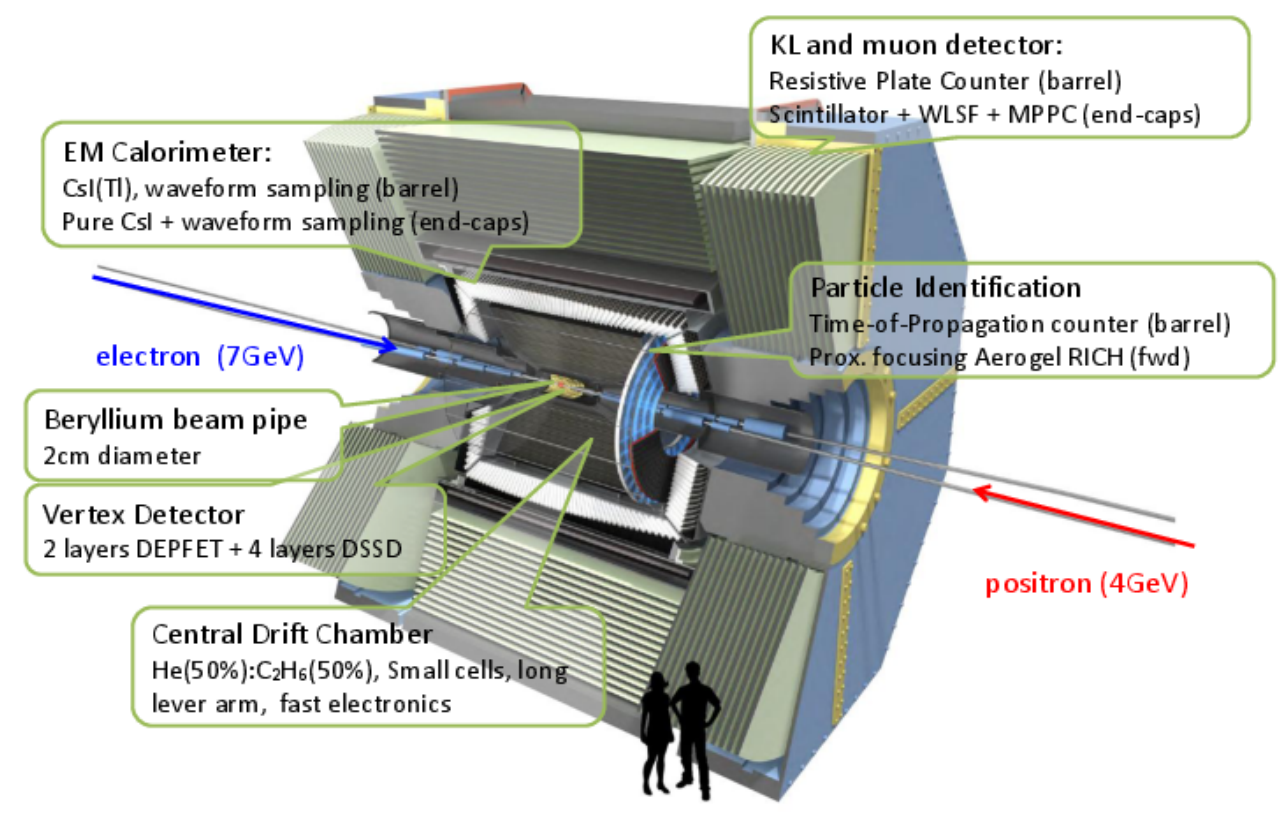

Figure 1: Belle II Detector

The Belle II detector is an upgraded general purpose spectrometer designed to collect data of electron-positron collisions provided by SuperKEKB accelerator efficiently [2]. The detector is also designed to have better or in same cases at least the same performance under about 20 times higher beam background environment. Since rare decay searches and efficient background rejection will play key roles, good particle identification (PID) in such high luminosity environment is required. For this purpose, Belle PID system will be replaced with new detectors. In Belle experiment, PID is performed with Time Of Flight (TOF), Central Drift Camber (CDC), and threshold type Aerogel Cherenkov Counter (ACC) [3]. These sub-detectors provide almost independent measurements and have a specifically good Kaon identification in different momentum regions. However, the effective momentum range is not wide enough for all particles from various decays. In Belle II experiment, two Ring Imaging CHerenkov (RICH) type systems as shown in Fig. 11 cover the whole momentum range. By using a barrel-shaped array of Time Of Propagation (TOP) counters and a proximity focusing Aerogel Ring Imaging CHerenkov (ARICH) detector in forward endcap region, we aim to have good $K / \pi$ separation power in momentum range up to about $4 \mathrm{GeV} / \mathrm{c}$. The Belle II detector 
also has an electromagnetic calorimeter (ECL) comprised of CsI scintillator crystals located outside of TOP counter and $K_{L}^{0}-\mu$ detector (KLM) which consists of an alternating sandwich of $4.7-\mathrm{cm}$ thick iron plates, and glass-electrode Resistive Plate Chambers (RPCs) and plastic scintillators located outside the superconducting solenoid. In this paper, the construction status of Belle II PID systems is briefly described.

\section{TOP counter}

TOP counter is a compact totally internally reflecting Cherenkov ring imaging detector for charged particle identification [4, 5, 6. The TOP counter consists of $2.7 \mathrm{~m}$ long high quality quartz radiator, a focusing spherical mirror at one end, and an array of high time resolution and position sensitive photo-detectors (Hamamatsu SL10 16ch Micro Channel Plate(MCP)-PMTs) at the other end (see Fig. 2). Cherenkov photons emitted by charged particle passing through the quartz radiator are bounced off $>100$ times at the quartz surface and detected with MCP-PMTs finally. Chromatic dispersion is minimized by using the focusing mirror and wavelength cut filters in front of MCP-PMTs. Cherenkov ring imaging is performed with two hit coordinates and precise Time Of Propagation (TOP) measurement $(\sigma \sim 50 \mathrm{ps}$ ).

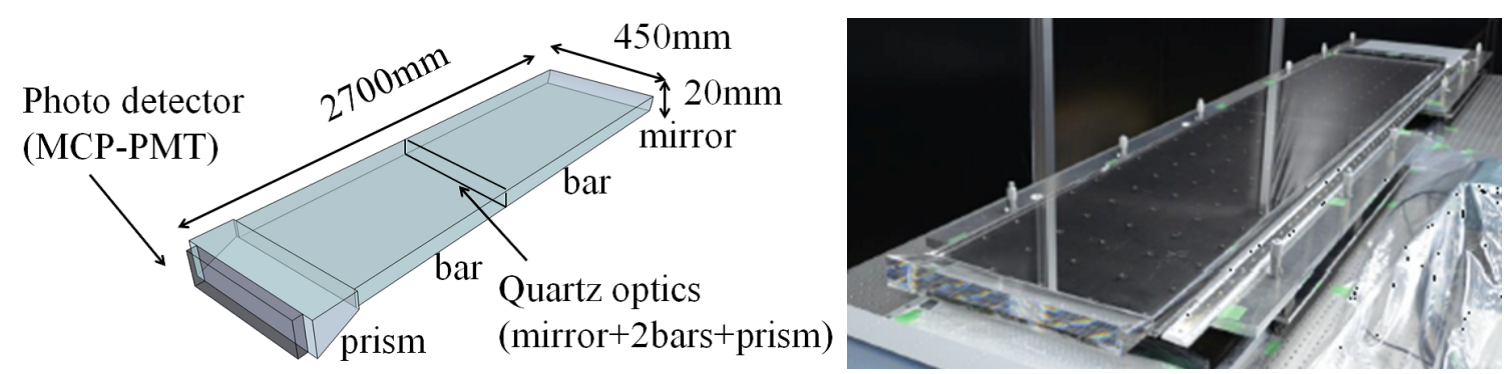

Figure 2: Overview of the Belle II TOP counter (left). The quartz radiator of the first full scale prototype assembled in the cleanroom at KEK (right).

Detector R \& D has been done in 2013 and a full scale prototype Belle II TOP counter was successfully assembled without any serious problems as shown in Fig. 2 The performance was evaluated at SPring-8 LEPS with $2 \mathrm{GeV} / \mathrm{c}$ position beam and cosmic muons [7, 8]. In these tests, beautiful Cherenkov ring images were obtained and the time distribution of each channel was in good agreement with Monte-Carlo expectation based on the specification parameters for the optical components and the photo-detectors as shown in Fig. 3 Number of detected Cherenkov photons was also obtained as expected. These results demonstrate that the expected performance of the TOP counter has been well understood. Using the beam test results, we have also checked the PID performance for various decay modes. The performance is adequate, for example, to achieve a measurement of $\left|V_{t d}\right| /\left|V_{t s}\right|$ with a few percent relative accuracy.

Detector construction is ongoing at KEK. Mass production of photo-detectors has already been completed and several tests are in progress. Front End Electronics (FEE) is based on high-speed waveform sampling ASICs developed by University of Hawaii. The final electronics production and validations will start soon. Procedures for acceptance test and assembly of the quartz radiator 

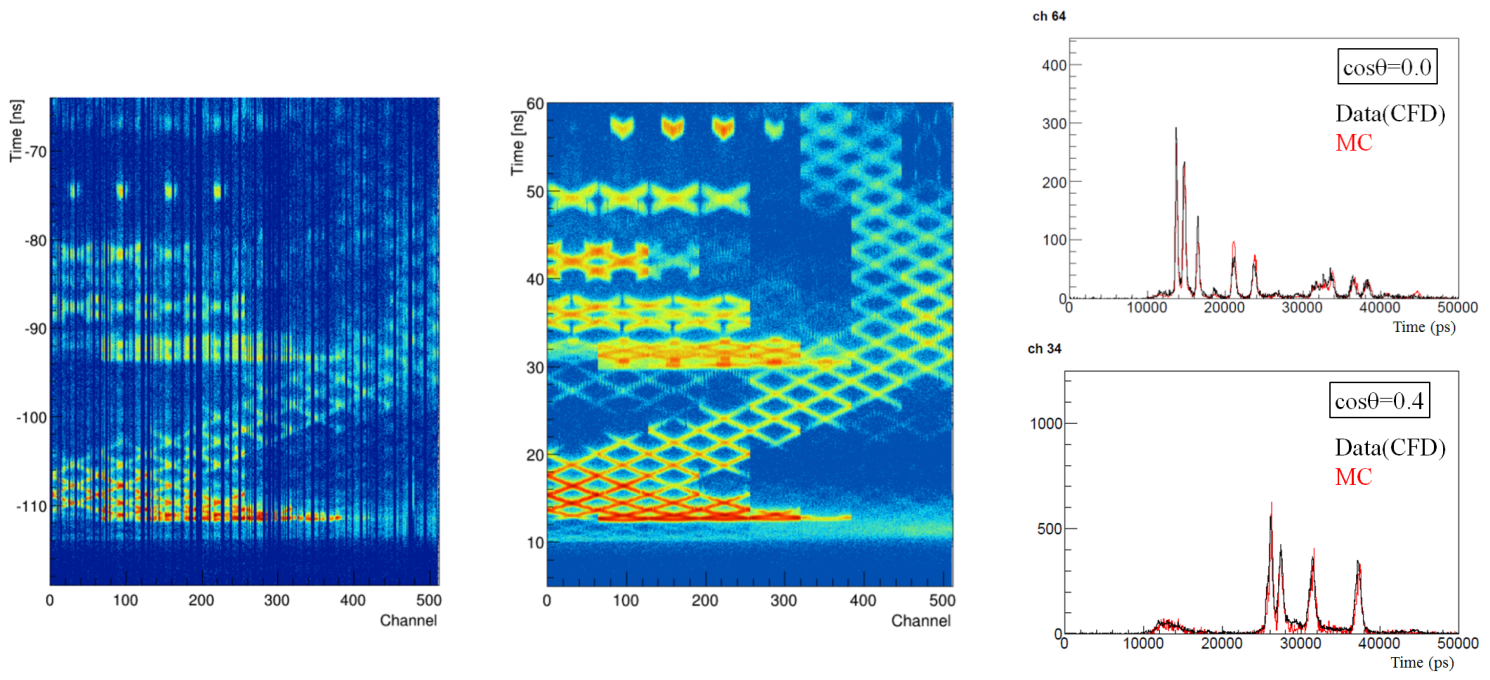

Figure 3: Cherenkov ring image obtained by data taken with IRS readout electronics (left) and Monte-Carlo simulation (center) as a function of PMT channel number. The beam data was taken with normal incident condition. Right panels show typical time distributions taken with backup CFD readout electronics.

is well established[9. Mass production of the precision optical components is now under way. The 1st Belle II TOP counter has been successfully assembled at KEK in October 2014. Mass production will start early in 2015.

\section{ARICH}

ARICH is the Belle II forward PID detector which identifies charged particle by difference of Cherenkov angle emitted in aerogel radiator[10]. Aerogel radiator consists of 2 layers with different refractive indices and proximity focusing technique is used due to limited space between CDC and ECL (see Fig. 4). 209 aerogel tiles $(n=1.045)$ and 240 tiles $(n=1.055)$ have been successfully produced. Test measurements are currently ongoing. 420 of Hamamatsu $5 \mathrm{~mm}$ pitch pixelated 144-channel Hybrid Avalanche Photo Detector (HAPD) will be used as photo-detectors. The mass production has been started in 2012. Until now $\sim 250 / 450$ HAPDs have already been delivered and $\sim 180$ HAPDs passed acceptance tests. Readout electronics is in good shape.

Beam tests were carried out with hadron beam at SPS in CERN and electron beam at DESY to evaluate the performance of the prototype ARICH. Simple performance estimation from cumulative Cherenkov angle distribution shows good Cherenkov angle resolution $\left(\Delta \theta_{c} \sim 14 \mathrm{mrad}\right)$ and large enough number of photons detected $(N \cdot p e \sim 11.4)$ as shown in Fig. 4 This beam test performance demonstrates the good $K / \pi$ separation power $(5.5 \sigma)$ to meet our requirements from physics motivation.

Event reconstruction software for TOP counter and ARICH has been developed. $K / \pi$ separation power of Belle II PID systems has been studied with Monte-Carlo samples and beam test performance. Using TOP counter, ARICH, and CDC (dE/dx) information, the estimated $K$ efficiency and $\pi$ fake rates are $94 \%$ and $4 \%$ respectively[13]. 

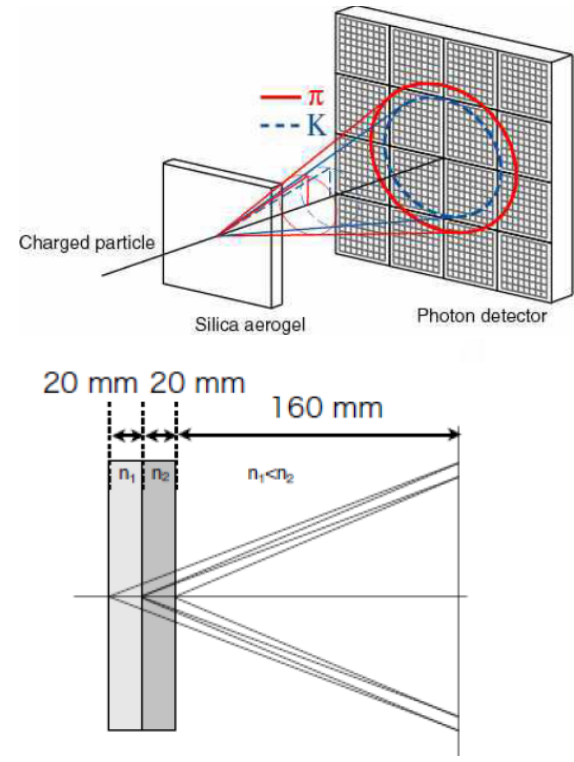
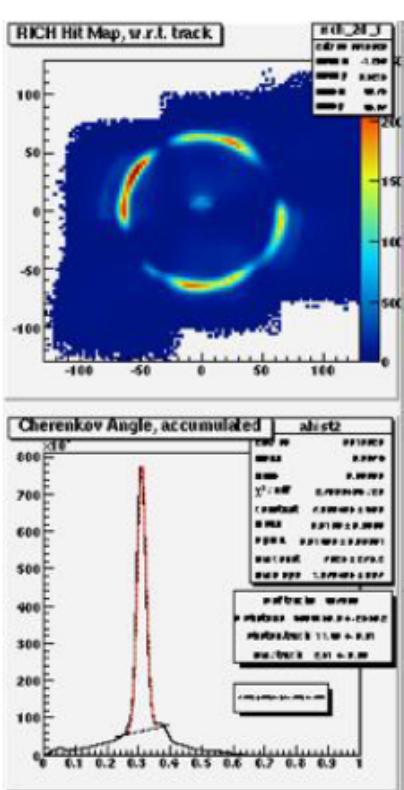

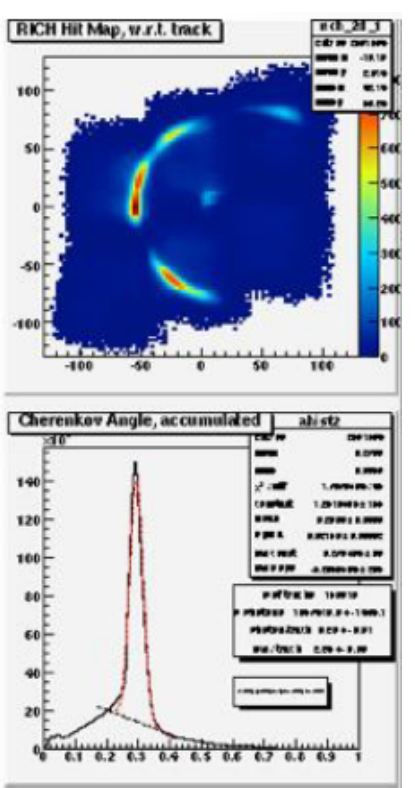

Figure 4: ARICH: Schematic illustration of the detection principle (left panel). Reconstructed Cherenkov rings produced by electron in the beam test data (right panel)

\section{ECL}

Belle electromagnetic calorimeter (ECL) is being upgraded. Belle's barrel CsI(Tl) crystals are reused, and new electronics is introduced with $2 \mathrm{MHz}$ waveform sampling to compensate for the larger beam related backgrounds and the long decay time of CsI(Tl). All ECL channels with new electronics have been tested and cosmic test will start soon[11].

In the endcap region, Belle endcap ECL crystals will also be reused with new preamplifiers and readout electronics at the first stage of the Belle II experiment. The new preamplifiers and readout electronics have been tested with the crystals. They will be installed in 2015. Some endcap ECL upgrade options are now under investigation. One of the main options is to replace $\mathrm{CsI}(\mathrm{Tl})$ with pure CsI for faster performance and better radiation hardness. Several photo-detectors options for pure CsI are also being studied.

\section{KLM}

Belle endcap $K_{L}^{0}-\mu$ detector (EKLM) is being renewed[12]. Resistive Plate Counters (RPCs) for EKLM are being replaced with scintillators to handle higher neutron backgrounds. New forward endcap sectors have been installed in April 2014 and backward sectors are being installed. Barrel $K_{L}^{0}-\mu$ detector (BKLM) has been upgraded too. Two inner layers of Resistive Plate Counters (RPCs) for BKLM have also been replaced by scintillator strips. Post integration test will be done with new Front End Electronics in 2014. Muon identification software has also been developed. Muon tracks are identified using KLM information and the performance will be tested with cosmic muons. 


\section{Summary}

The Belle / KEKB is being upgraded to Belle II / SuperKEKB with the goal of probing new physics beyond the Standard Model (SM). Construction of the Belle II detector is going on now. Development of new barrel PID system (TOP counter) is being finalized. The 1st full scale prototype has been successfully assembled in 2013 and the performance was evaluated well at SPring8/LEPS. Detector construction will start in October 2014. Construction of forward endcap PID detector (ARICH) is also in good shape. Results of beam tests with prototype ARICH satisfy requirements from physics motivation. ARICH installation will be done in 2015. Upgrade of $K_{L}^{0}-\mu$ detector (KLM) and electromagnetic calorimeter (ECL) is proceeding according to schedule. Post-integration test and software development are also going well. Detector construction will be completed in 2016 and Physics Run will start in 2017.

\section{Acknowledgements}

I would like to thank Belle II PID group for useful suggestions and discussions. Detector R \& $\mathrm{D}$ and construction of Belle II TOP counter were supported by MEXT Grant-in-Aid for Scientific Research on Innovative Areas "Elucidation of New Hadrons with a Variety of Flavors" and Grantin-Aid for Young Scientists (B).

\section{References}

[1] Belle, A. Abshian et al., Nucl. Instr. and Meth. A 479, (202) 117.

[2] T. Abe et al., arXiv:1011.0352.

[3] E. Nakano et al., Nucl. Instr. and Meth. A 494, (202) 402.

[4] M. Akatsu et al., Nucl. Instr. and Meth A 440 (2000) 124.

[5] K. Inami et al., Nucl. Instr. and Meth. A 766 (2014) 5.

[6] K. Matsuoka et al., Nucl. Instr. and Meth A 766 (2014) 163.

[7] T. Nakano et al., Nucl. Phys. A 684 (2001) 71.

[8] T. Hayakawa et al., Nucl. Instr. and Meth. A 766 (2014) 255.

[9] B. Wang et al., Nucl. Instr. and Meth. A 766 (2014) 204.

[10] S. Nishida et al., Nucl. Instr. and Meth. A 766 (2014) 28.

[11] V. Aulchenko et al., JINST 9 C0914 (2014).

[12] A. Abashian et al., Nucl. Instr. and Meth. A 449 (2000) 112.

[13] M. Staric et al., Nucl. Instr. and Meth. A 766 (2014) 237. 